\title{
AA $\beta$ public-key cryptosystem - a practical implementation of the new asymmetric
}

\begin{abstract}
This paper aims to provide a practical implementation one of a probabilistic cipher proposed by M.R.K. Ariffin, M.A. Asbullah and N.A. Abu called as AA $\beta$. We provide details on designing and implementing the AA $\beta$ algorithm. Furthermore, to support our understanding by providing a statistical analysis of times taken to implement the key generation, encryption and decryption algorithm for the key sizes 3072, 6144, 9216 and 12288 bits for message spaces of $4 n$ where $n=512,1024,1536$ and 2048 bits. We show the working of the AA $\beta$ algorithm purely from a practical standpoint to justify if it is practically implementable even for large data sets operating on large key sizes.
\end{abstract}

Keyword: Probabilistic cipher; Public key cryptosystem; AA $\beta$ public key cryptosystem. 